\title{
Dispepsia ed Helicobacter pylori
}

\section{Giovanni Fornaciari}

III Divisione di Medicina e Gastroenterologia Arcispedale S. Maria Nuova Reggio Emilia

\section{SUMMARY}

The effect of Helicobacter pylori (HP) eradication on functional dyspepsia has been analysed in several clinical trials, including large, controlled and well-designed studies as well as small, flowed studies. The results of these studies indicate that HP infection does not play a major role in the aetiology of this disease and that HP eradication improves dyspeptic symptoms in no more than 15\% of patients as compared to placebo. From a practical point of view 15 patients need to be treated for one to benefit while, in duodenal ulcer, I.4 patient need to be treated for one to benefit. It remains to be elucidated if HP eradication in functional dyspepsia is useful to reduce the risk of developing organic dyspepsia (namely peptic ulcer) in functional dyspepsia. In uninvestigated dyspepsia the management of HP infection in primary care has been fully debated. Two therapeutics strategies have been proposed: test and scope and test and treat. The value of test and treat strategy over alternative strategies has been demonstrated in several decision analyses. HP test and scope increases costs in primary care without improving symptoms and saves only I5\% of endoscopies.

\section{RELAZIONE}

\section{Definizione}

La parola dispepsia deriva dal greco e significa cattiva digestione; negli anni più di dieci definizioni diverse dal 1960 sono comparse in letteratura in quanto si tratta, come precisato dagli autori, di una malattia sfuggente, mal definita e misconosciuta. In due successive Consensus Conference tenutesi a Roma è stata finalmente fornita una definizione accettata attualmente da tutti: dolore $o$ fastidio addominale persistente o ricorrente localizzato ai quadranti superiori dell'addome successivamente meglio precisato come localizzato prevalentemente in regione epigastrica. (1) Questo prevede la esclusione dei pazienti con malattia da reflusso gastro-esofageo e/o sindrome dell'intestino irritabile (e questo non è sempre agevole).

\section{Epidemiologia}

Si tratta di un problema di grande rilevanza clinica con una prevalenza che oscilla fra il 20 e il $40 \%$ della popolazione adulta ed un'incidenza dell' $1-8 \%$ annuo. Il 3\% delle visite del Medico di Medicina Generale, il 40\% delle visite specialistiche sono per dispepsia. In Gran Bretagna vengono eseguite più di 500.000 gastroscopie ogni anno e vennero spesi nel 1998 spesi 1.1 miliardi di sterline per questa patologia (2).

\section{Classificazione}

La classificazione comunemente accettata è la seguente:

- Dispepsia organica:

ulcera, tumori, esofagite, celiachia, angina abdominis, pancreatite, colelitiasi, diabete, malattie sistemiche (LES, amiloidosi) ecc.

- Dispepsia funzionale: nella quale è stata esclusa una delle cause sopra riportate

- Dispepsia non investigata: nella quale non sono stati ancora eseguiti accertamenti

\section{Sintomatologia}

I classici sintomi sono:

- Dolore o pirosi epigastrica

- Pesantezza o ripienezza post-prandiale

- Digestione laboriosa

- Gonfiore post-prandiale

- Nausea

- Conati e vomito: questi sintomi sono più raramente riportati nella dispepsia funzionale.

Alcuni sintomi, definiti d'allarme, devono essere riconosciuti in quanto possono essere indicativi per una forma organica e non funzionale:

- Età all'esordio superiore a 45 anni

- Dolore irradiato al dorso

- Dolore notturno

- Vomito e/o disfagia progressiva

- Calo ponderale rapido

- Assunzione cronica di alcool o FANS

- Abitudine al fumo

\section{Classificazione della dispepsia funzionale}

La dispepsia funzionale viene classificata comunemente in tre sottotipi:

- Dispepsia simil-ulcerosa: prevalenza di dolore e/o pirosi epigastrica

- Dispepsia simil-motoria: prevalenza di "discomfort" senza dolore

- Dispepsia non classificabile In precedenza veniva classificato anche un gruppo della dispepsia simil-reflusso che attualmente non è più accettato

\section{Fisiopatologia}

Si tratta di un meccanismo complesso e non del tutto chiarito nel quale giocano un ruolo fondamentale diversi fattori: 
- Secrezione acida

- Sensibilità viscerale

- Alterazioni della motilità gastrica

- Helicobacter pylori

Secrezione acida: circa il 20\% dei casi di dispepsia funzionale dovrebbe essere sostenuta da alterata secrezione acida. Ne è prova il rilievo che l'instillazione di acido provoca i sintomi della malattia e che la terapia con anti-secretivi è efficace nei pazienti con dispepsia simil-ulcerosa anche se non lo è nei pazienti con forma similmotoria (3)

Sensibilità viscerale: esiste una ridotta soglia alla stimolazione meccanica e chimica del fondo gastrico ed un'alterata risposta delle afferenze nervose simpatiche o vagali. Infatti è documentata una risposta a farmaci modulatori della sensibilità viscerale (fedotozina, antagonisti della serotonina ecc.) in studi preliminari (4)

Alterazioni della motilità gastrica: è stata dimostrata ipomobilità antrale e rallentato svuotamento gastrico oltre ad alterazione della capacità di accomodazione del fondo gastrico in base ad alterazione del sistema vagale. Sono probabilmente in gioco meccanismi ormonali (4)

Helicobacter pylori (HP) i primi studi di Marshall (5) evidenziarono la comparsa di dispepsia dopo ingestione sperimentale di HP e regressione dopo l'eradicazione. L'HP induce aumento della gastrina con incremento della secrezione acida che si risolve con l'eradicazione. L'associazione fra infezione da HP e gastrite cronica è comunemente accettata ma il ruolo della gastrite cronica nel causare sintomi dispeptici è molto meno chiaro. I rapporti fra HP e motilità digestiva sono complessi e di difficile interpretazione. Non sembra esistere rapporto fra HP ed aumentata sensibilità viscerale (6)

\section{Prevalenza HP nella dispepsia funzionale}

Sono stati pubblicati diversi studi epidemiologici con risultati contrastanti: negli studi di popolazione vi è un apparente ruolo dell'HP in particolare sui sintomi pirosi e dolore ma si devono registrare diversi bias: elevata prevalenza HP nei controlli e controlli non sempre "well matched". Una metaanalisi: ha dimostrato una odds ratio di 2.3 (1.92.7) (7). È stato calcolato che i pazienti con infezione da HP hanno un rischio aumentato del $22 \%$ di presentare dispepsia organica o funzionale (8).

\section{Efficacia del trattamento nel controllo dei sin- tomi della dispepsia funzionale}

Molti studi sono stati pubblicati sull'argomento con risultati contrastanti; gli studi avevano spesso un disegno metodologico inadeguato ed una variabile risposta al placebo (13-73\%). Peraltro, anche se è difficile la valutazione dei sintomi, le meta-analisi Anche le meta-analisi sono problematiche ma sembrano dimostrare in sostanza un effetto positivo. D'altra anche nei trials correttamente disegnati il risultato è dubbio; le conclusioni che si possono trarre sono che l'eradicazione dell'HP può essere utile in un gruppo di pazienti (probabilmente il 20\%) con dispepsia funzionale ma che è difficile identificare quali pazienti si gioveranno del trattamento (9-10).

I due studi più recenti ed eseguiti con metodologia simile e con esclusione dei pazienti affetti da reflusso gastro-esofageo (Studio ORCHID - Studio OCAY) hanno messo a confronto la terapia eradicante (omeprazolo, amoxicillina e claritromicina per 7 giorni) con il placebo. È stato eseguito un follow up a un anno con controllo endoscopico. Non sono emerse differenze significative complessive $\mathrm{ma}$, anche in questo caso, si è notato un beneficio per la dispepsia "ulcer like" o "reflux like" (11-12). Nella recente analisi della Cochrane Library la riduzione del rischio relativo risulta essere del 9\% (CI 5-14\%); in "pratica 15 pazienti (CI 10-28) con dispepsia funzionale devono essere trattati per ottenere beneficio in un paziente mentre nell'ulcera duodenale 1.4 pazienti devono essere trattati per ottenere beneficio in un paziente (13).

\section{Prevenzione dell'insorgenza di dispepsia organica}

Uno dei vantaggi che si potrebbero ottenere, mediante il trattamento dell'HP nella dispepsia funzionale, risiede nel rilievo che l'HP potrebbe predisporre all'insorgenza di dispepsia organica ed in particolare di ulcera peptica. In tre trias $(11,12$, 14) sono segnalate ulcere peptiche nel $4.1 \%$ dei pazienti trattati con placebo; il rischio di sviluppare ulcera peptica sembra più elevato nella dispepsia funzionale simil-ulcerosa. Il risultato della terapia eradicante può quindi essere legato alla riduzione del rischio di sviluppare, negli anni successivi, una ulcera peptica. Occorre però segnalare che la conoscenza del test per l'HP può influenzare la sintomatologia riferita dal paziente (10)

\section{Gestione pratica del paziente}

Tutto quanto sopra descritto porta ad individuare due possibili strategie che sono state ampiamente discusse e studiate negli ultimi tempi:

Test and treat: test non invasivo per HP a tutti i pazienti e terapia eradicante sui pazienti HP positivi. Gastroscopia in caso di mancata risposta

Test and scope: test non invasivo per HP a tutti i pazienti e gastroscopia per quelli HP positivi, terapia empirica per quelli HP negativi

Quest'ultima strategia non si è rivelata vantaggiosa in quanto aumenta i costi senza vantaggi in ter- 
mini di riduzione dei sintomi e di miglioramento della qualità della vita. Inoltre riduce solo del 15$20 \%$ il numero delle gastroscopie richieste (15).

Per quanto attiene alla strategia "test and treat", invece, quattro trials randomizzati hanno dimostrato pari efficacia rispetto alla endoscopia immediata. Inoltre la strategia risulta cost-effective rispetto alla endoscopia immediata, alla terapia empirica ed al "test and scope" con una qualità della vita simile (16). Il rationale di questa strategia è basato sul rilievo che l'HP si associa a gastrite cronica e che l'eradicazione comporta un miglioramento istologico; inoltre l'eradicazione determina la guarigione della maggior parte delle ulcere e potrebbe portare a riduzione dell'incidenza di cancro gastrico. Il test and treat risulta vantaggioso in termini di costo-beneficio in particolare nella dispepsia funzionale simil-ulcerosa anche se i pazienti sembrano meno "rassicurati" non essendo sottoposti a gastroscopia. Inoltre questa strategia potrebbe portare a antibioticoresistenza dell'HP ed a aumento della malattia da reflusso gastro-esofageo (10).

La recente Consensus Conference di Maastricht conferma che la strategia "test and treat" è indicata in pazienti al di sotto dei 45 anni, in assenza di "sintomi d'allarme". L'eradicazione dell'HP è "considerata" appropriata in caso di dispepsia funzionale in quanto ottiene un miglioramento, a lungo termine, in un sottogruppo di pazienti (17)

\section{BIBLIOGRAFIA}

1. Talley NJ et al. Functional dyspepsia: a classification with guidelines for management. Gastroenterol. Int 1991; 4: 145-60

2. Talley NJ et al. AGA technical review: evaluation of dyspepsia. Gastroenterology 1998; 114: 582-595

3. Samson M et al. Abnormal clearance of exogenous acid and increase acid sensitivity of the proximal duodenum in dyspeptic patients. Gastroenterology 1999; 116: 761-762

4. Stanghellini $\mathrm{V}$ et al. Risk indicators of delayed gastric emptying of solids in patients with functional dyspepsia. Gastroenterology 1996; 110: 1036-1041

5. Marshall BJ et al. Pyloric Campylobacter infection and gastroduodenal disease. Med. J. Aust. 1985; 142: 439-4444

6. Armstrong D. Helicobacter pylori infection and dyspepsia. Scand. J. Gastroenterol. 1996; 31 (suppl. 215): 38-47

7. Bazzoli $\mathrm{F}$ et al. Helicobacter pylori and functional dyspepsia: review of previous studies and commentary on new data. Gut 2002; 50 (suppl. IV): 33-35

8. Bytzer $\mathrm{P}$ et al.

Dyspepsia. Ann. Int. Med. 2001; 134: 815-822

9. Talley NJ et al. Helicobacter pylori and non ulcer dyspesia. Aliment. Pharmacol. 2002; 16 (suppl. 1) : 58-65

10. Bazzoli $\mathrm{F}$ et al. Treatment of Helicobacter pylori infection. Indications and regimens: an update. Digest. Liver Dis. 2002 ; 34 : 70-83

11. Talley NJ et al. Eradication of Helicobacter pylori in functional dyspepsia : randomised controlled trial with 12 months follow up. The ORCHID study. BMJ 1999; 316: 833-877

12. Blum AL et al. Lack of effect of treating Helicobacter pylori infection in patients with nonulcer dyspepsia. N. Engl. J. Med. 1998; 329: 1875-1881

13. Moayyedi $\mathrm{P}$ et al. Eradication of Helicobacter pylori for non ulcer dyspepsia (Cochrane Review) 2003; issue 1

14. Mc Coll KE ed al. Eradication of Helicobacter pylori in functional dyspepsia. BMJ 1999; 319: 451-452

15. Gisbert JP et al. Helicobacter pylori "test and scope" strategy for dyspeptic patients. Is it useful and safe? Dig. Liver Dis. 2001; 33: 539-545

16. Moayyedi P. Helicobacter pylori test and treat strategy for young dyspeptic patients: new data. Gut 2002, 50 (suppl. IV): 47-50

17. Malfertheiner $P$ et al. Current concepts in the management of Helicobacter pylori infection- the Maastricht 2-2000 Consensus Report. Aliment. Pharmacol. Ther. 2002; 16: 167-180 